\title{
Experimental Study on Biohazard Missile Detection and Automatic Destroy System
}

\author{
Manjula B K, Venkata Sumana CH and Dr. Latha B M \\ Department of Electronics and Communication Engineering, GM Institute of Technology, Davangere, India
}

\begin{abstract}
This suggested device is equipped with the aid of ultrasonic sensors to detect the target (missile, aircraft, drones etc.) approaching and automatically kill it. The ultrasonic transducer is rotated at 360 degrees and consists of a transmitter and a receiver. The sound waves are emitted from the transducer and the transducer receives them again from the target. The ultrasonic transducer is linked to the microcontroller of the PIC. When the target is recognized within the detection range, the signal is received by the microcontroller. On the microcontroller, the software assigned will move the launching application to the degree detected and aim towards the target. This proposed device uses an ultrasonic module connected to the 8051 family of microcontrollers. An ultrasonic transducer consisting of a receiver and a transmitter is used. The waves transmitted are reflected back from the object and are again received by the transducer. The cumulative time taken from sending to receiving the waves is determined by taking the sound velocity into account. The distance is then determined by a program running on the microcontroller and displayed via wireless communication on a liquid crystal display screen interconnected to the microcontroller. The circuit is used to receive $40 \mathrm{KHz}$ reflected signals from the missile object, to feed them to the microcontroller program and to turn on the required load while the microcontroller is running the program. When the microcontroller receives the signal from the ultrasonic receiver, the door gun is triggered through a transistor or relay by triggering the MOSFET gate. The sensor is mounted on the antenna and is rotated and operated by a 360 degree stepper motor.The program will move the launcher to the nearest detected target and fire if there is any target inside the detection range.
\end{abstract}

KEY WORDS: MICROCONTROLLER; ULTRASONIC SENOR; PROXIMITY DETECTOR; STEPPER MOTOR; MPLAB IDE SOFTWARE; DRIVER IC.

\section{INTRODUCTION}

War is a hybrid conflict of governments, nations, ethnic and social groups that is carried out. This autonomous, battery or air conditioning powered transmitter and recipient area ultrasonic proximity finder uses a few synchronized ultrasonic fired transducers, each operating at around $40 \mathrm{kHz}$ (Puttamadappa \&t Parameshachari 2019; Kumar \&t Furuhashi 2017; Kumar \& Furuhashi, 2018). A

\section{ARTICLE INFORMATION}

*Corresponding Author: manjulabk@gmit.ac.in

Received 10th Oct 2020 Accepted after revision 25th Dec 2020

Print ISSN: 0974-6455 Online ISSN: 2321-4007 CODEN: BBRCBA

Thomson Reuters ISI Web of Science Clarivate Analytics USA and Crossref Indexed Journal

\section{Clarivate
Analytics}

NAAS Journal Score 2020 (4.31)

A Society of Science and Nature Publication,

Bhopal India 2020. All rights reserved.

Online Contents Available at: http//www.bbrc.in/

Doi: http://dx.doi.org/10.21786/bbrc/13.13/52 missile, also referred to as a guided missile, is a guided airborne range weapon capable of self-propelled flight, normally by a jet engine or rocket engine in modern language (Guo et al. 2018; Ming et al. 2019; Ming et al. 2019; Garcia et al. 2018). There are four system elements for missiles: targeting/guidance system, flight system, engine and warhead. Missiles come in forms tailored for various purposes: ballistic surface-to-surface and air-to-surface missiles, cruise, anti-ship, anti-tank, surface-to-air (and anti-ballistic), air-to-air missiles, and anti-satellite weapons (Chai et al. 2019; Hong et al. 2020; Wang et al. 2019; Zhang et al. 2020).

This circuit is used to get $40 \mathrm{KHz}$ reflected signals from the rocket to a program to the microcontroller to turn on the required weight while the program is executed at the end of the microcontroller to deal with that. When the microcontroller receives the sign from the ultrasonic recipient, the gateway weapon begins by setting off the

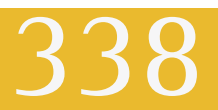


MOSFET entrance through a semiconductor. The force is flexibly composed of a 230/12V stage-down converter, which decreases the voltage to $12 \mathrm{~V}$ air conditioning. This is altered to DC at that stage using an Extension rectifier. Using a capacitive channel, the waves are then evacuated and then controlled to $+5 \mathrm{~V}$ using a voltage controller 7805 that is necessary for the operation of the microcontroller $\&$ various segments. OpAmps are used by the receiving ultrasonic transducer sent by the communicating one to improve the fragile signs obtained upon reflection from the obstruction, to turn on the required burden while the program is executed at the end of the microcontroller.

Motivation: The use of new technology in the field of Machine Learning is growing rapidly. Object detection is the core component of the missile detection and destruction scheme. We will apply the ultrasonic sensorbased detection method for this reason. This will give us the exact distance of the object from the unit of destruction. The robotic wheel will work according to the distance measured. We will use the Bluetooth module for the operation of the robotic wheel signal from the Android app and for contact between the Android app and the robot wheel. An antenna will be fitted with the sensor circuitry. The combination of the ultrasonic sensor and the stepper motor will make up the antenna. To give the control signal, i.e. to travel forward, backward, left or right, the Bluetooth software will be used. The microcontroller will be the key component of this device.

We use MP LAB tools for coding this controller. The circuit is used to receive $40 \mathrm{KHz}$ reflected signals from the missile object, to feed them to the microcontroller program and to turn on the required load while the microcontroller is running the program. When the microcontroller receives the signal from the ultrasonic receiver, the door gun is triggered through a transistor or relay by triggering the MOSFET gate. The sensor is mounted on the antenna and is rotated and operated by a 360 degree stepper motor. The program will move the launcher to the nearest detected target and fire if there is any target inside the detection range. The venture consists of an ultrasonic transmitter and a collector, each of which operates at a frequency of $40 \mathrm{kHz}$. Here, together with a stepper motor equipped with an ultrasonic sensor, a robotic platform is used to automatically locate and target a stationary target, move the target to a pre-defined range and successfully fire a laser. Let's summarize the key progress of the research and point out potential directions for future work.

Objectives: The motivation behind this undertaking is to plan and develop programmed rocket recognition and annihilating framework. This framework is intended to recognize the objective (rocket) moving in numerous bearings. The objective pulverizing framework moves naturally toward rocket and fires it after fixing the objective. This rocket location and self-governing propelling system undertaking is amended by following the Ultrasonic transceiver knowledge to focus on a rocket object in three pivot turns.

1. Checking the moving objective.

2. Constant goal observation.

3. Works in any lighting conditions.

4. Programmed assault targets.

5. Robot power with the aid of RF TX and RF RX.

6. Checking that the target is hostile or not.

7. If hostile automatic targeting it by laser.

8. If not hostile moves further.

9. This all features in moving bot.

Literature Survey: Customized Rocket Pointer Using Ultrasonic Proximity Locater. (April 2016) Narayan Thakkar et al. The proposed device uses the 8051 Microcontroller as a central control mechanism to send control requests to concentrate on the structure through laser methods to ambush the target (rocket). The Intel MCS-51 (mostly 8051) is a Complex Direction Set Enrolling (CISC) Direction Set within Harvard, a single chip microcontroller course of action developed by Intel for use in embedded systems.

Microcontroller Based Rocket Recognizable proof and Destroying Structure. (July 2014) et.al. The proposed paper outlines that this endeavor includes an astute sonar-based article that uses a worldwide locating device and a DC furnished motor-driven ending unit interfaced with a control unit based on a microcontroller. Instead of the IR sensor, the ultrasonic sensor is sponsored, since the ultrasonic sensors can cover gigantic divisions and can discern the center under all lighting conditions. As a control unit, the Atmel 89c52 microcontroller is used. The control unit sends requests to the terminating unit to pound the target as the aim (rocket) is recognized. Microcontroller programming is performed using the embedded 'c' language.

Rocket Discovery and Programmed Decimate Framework (May 2017) Sachin Gardi et al. This proposed framework uses an ultrasonic sensor module linked to the AVR family of microcontrollers. It uses an ultrasonic transducer consisting of a transmitter and a collector. With the aid of recycling wire and stepper engine pivot reception apparatus 360 degrees, the ultrasonic transducer will detect the article on the off chance that any item recognized will appear on the LCD show at that point. For moving equipment with radio wire, the DC engine was used. The ATmega32 control unit is a low-power, 8-piece CMOS microcontroller based on the upgraded RISC engineering of the AVR.

Rocket Discovery and Programmed Obliterate Framework (June 2015) Ms.Palwe Pooja et al. A rocket position and auto crush structure on a robot stage are used in this suggested framework. For stacking the Mounted C program, an ATmega16 microcontroller is used. Coffey, et al. (Coffey et al. 2019) applied DeepSqueak method for the detection and classification of the object automatically, rapidly, and reliably using cutting-edge regional convolutional neural network architecture. The analysis shows that Deep Squeak method has higher 
recall, reduced false positive rate and dramatically reduces the analysis time.

\section{METHODOLOGY}

Project description: The identification of the rocket and the programmed destruction framework using 8-piece microcontrollers are shown in the figure. The PIC16F877A is a low-power, tip-top 8-piece CMOS microcomputer with programmable and erasable $4 \mathrm{~K}$ bytes of Glint read-only memory (PEROM). The system is made using Atmel's unique memory production of high thickness and is impeccable with the path set and pinout of the MCS-51 business standard. The on-chip Streak allows the memory of the program to be reinvented in the system or by a normal software engineer who is not insecure. The PIC16F877A is an incredible microcomputer that offers a deeply adaptable and financially savvy arrangement by combining an adaptable 8-piece CPU with Streak on a solid chip. With standard features, the PIC16F877A gives the go: Flicker 4K bytes, Hammer 128 bytes, 32 I/0 row, two 16-piece clock/counters, a structured fivevector two-level meddle, a successive full duplex port, and oscillator and clock equipment on-chip.

The PIC16F877A is also structured with stable rationale for action down to zero repetition and supports two of the most loved power saving modes of programming. The Dormant Mode prevents the CPU while enabling the structure to continue operating with the Hammer, clock/counters, consecutive port and barge. The Shut down Mode saves the material of the Crush, but freezes the oscillator before the corresponding hardware resets, crippling all other chip limits. This section contains a detailed block diagram and a summary of the block diagram with regard to all the main project modules. With the support of antenna and stepper motor rotate antenna 360 degree, the object will be sensed in the given diagram ultrasonic transducer, if any object detected it will be shown on the LCD monitor.DC motor has been used for moving antenna hardware with right, left, upward downward motion it requires 5 volt power supply.

Any projectile thrown at a target with the intention of hitting it is essentially a missile. A stone thrown at a bird is, thus, a projectile. By using its reasoning capacity, the bird will evade the missile (the stone) by shifting either to the left, right, top or bottom with respect to the missile's flight path (trajectory). The missile was thus unsuccessful in its aim of reaching the bird in this situation (the target). Now, if the stone is also given any knowledge and rapid reaction to move with respect to the bird, to resolve targeting mistakes and the evasive actions of the bird and strike it accurately, the stone now becomes a guided missile. The key technologies of guided missiles are the integration of the energy source in a missile to provide the necessary force for its movement (propulsion), intelligence to go in the correct direction (guidance) and successful manoeuvring (control). They help to make a missile unique to a target, that is to say, to decide a missile's size, range and state of motion.
The Dormant Mode stops the CPU while allowing the structure to continue running on the hammer, clock/ counters, consecutive port and barge.

The Shut down mode saves the material of the Crush, but freezes the oscillator before the corresponding hardware resets, crippling all other chip limits.DC motor has been used for moving antenna hardware with right, left, upward downward motion .it requires 5 volt power supply. Any projectile thrown at a target with the intention of hitting it is essentially a missile. A stone thrown at a bird is, thus, a projectile. By using its reasoning capacity, the bird will evade the missile (the stone) by shifting either to the left, right, top or bottom with respect to the missile's flight path (trajectory). The missile was thus unsuccessful in its aim of reaching the bird in this situation (the target).

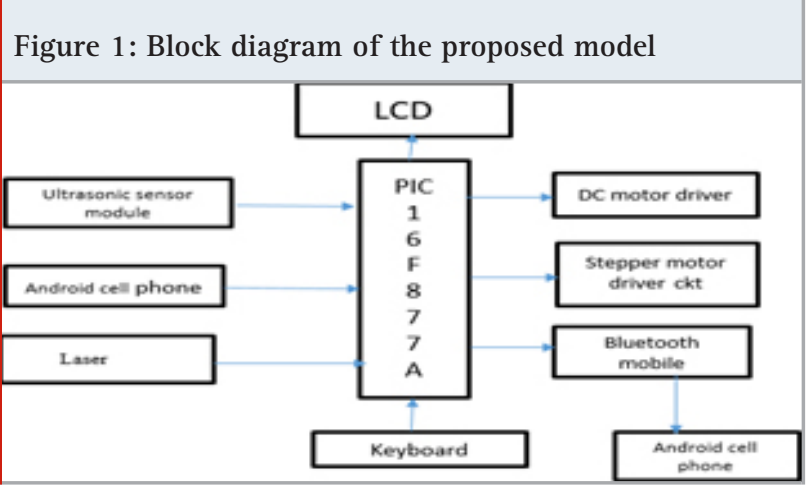

Figure 2: Prototype of Microcontroller based Missile Detector.

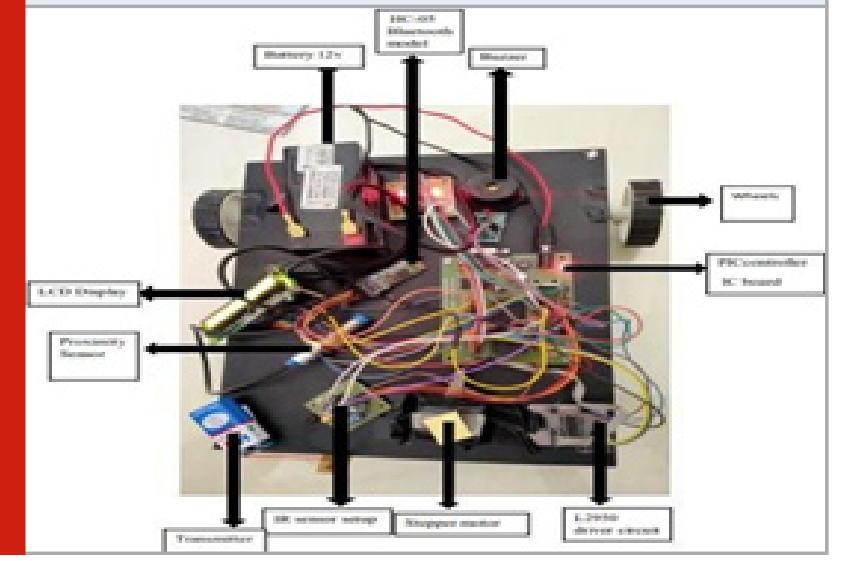

This proposed system incorporates a fully automated system which will save precious time as a result of this. The ultrasonic sensors can also detect the drone's. The controller is interfaced with the ultrasonic sensor here and it is rotated 360 degrees and continues to transmit and receive the sound waves. The launching machine will transform to the degree of the detected target and shoots when the object is detected. The figure turned up. The PIC16F877A is a low-power, tip-top 8-piece CMOS microcomputer with programmable and erasable $4 \mathrm{~K}$ bytes of Glint read-only memory (PEROM). The system is manufactured using Atmel's unique memory production 
of high thickness and is impeccable with the direction set and pinout of the business standard MCS-51.

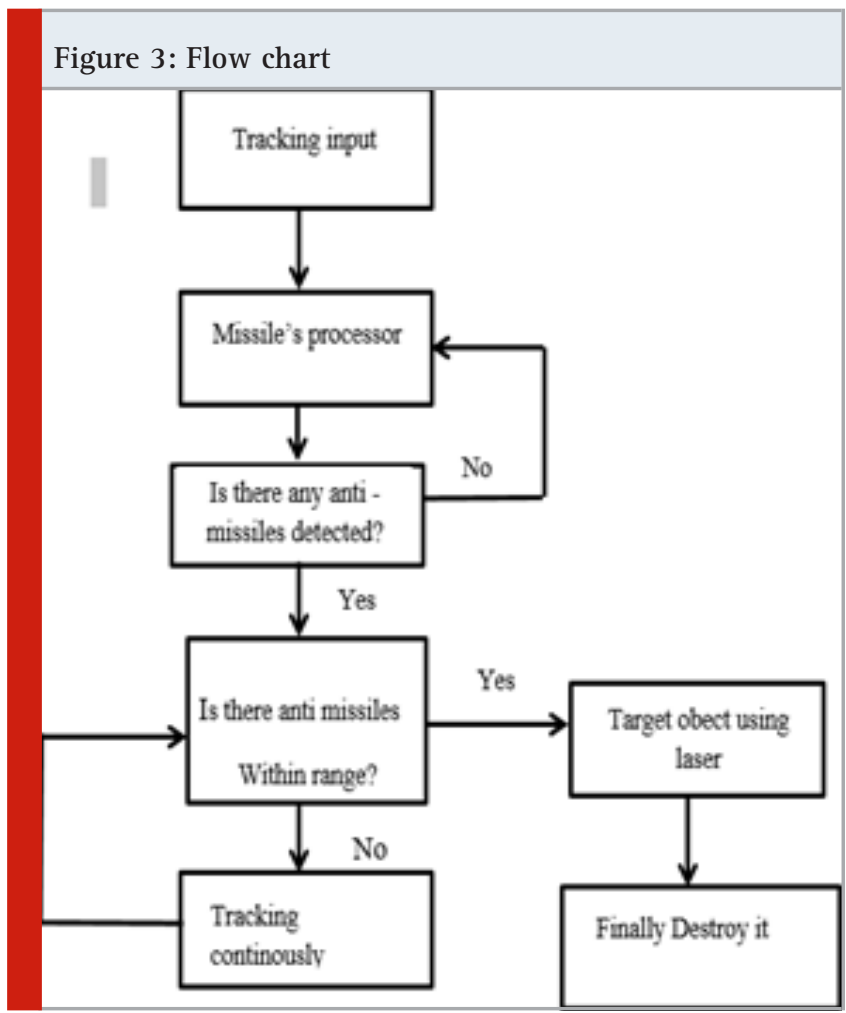

Figure 4: MP LAB IDE Software

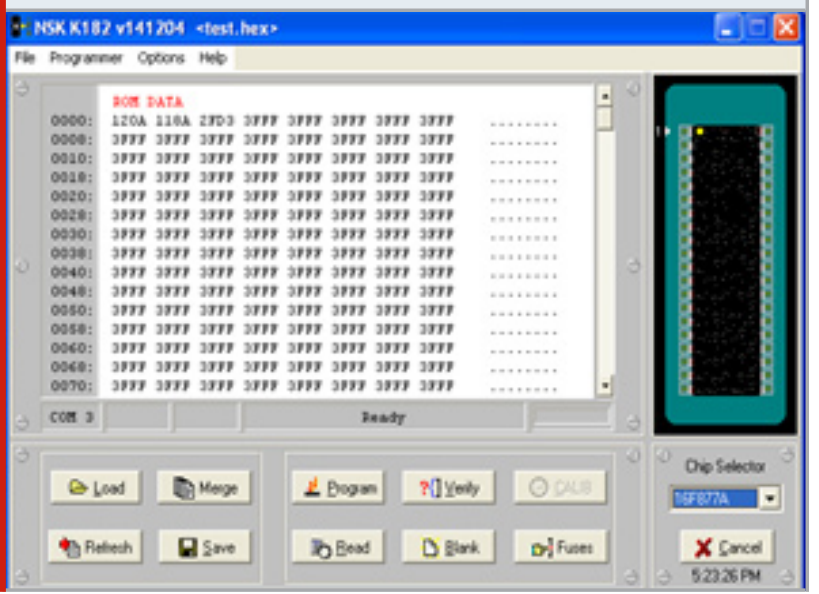

The purpose behind this undertaking is to design and create modified rocket revelation and smashing structure. This system is expected to distinguish target (rocket) moving in various course. The target obliterating structure moves subsequently toward rocket and flames it in the wake of fixing the goal. This structure involves a savvy sonar based thing worldwide situating system that endlessly screens the goal. In this endeavor we are using ultrasonic radar system and DC furnished motor driven ending unit cover stood up to with scaled down scale regulator based control unit.We lean towards the IR sensor ultrasonic marker, as the ultrasonic sensors cover greater separation detection and can perceive center in all lighting conditions (day or night). The programming of littler scope regulator is done using introduced 'c' language.

\section{Working setup}

- The framework regulators used to identify rocket objects.

- An ultrasonic indicator with a transmitter and beneficiary is utilized. They produce sound waves that communicate and when it thinks about it falls the transducer itself.

- The absolute time required to send the waves and get it determined the speed of sound.

- Then the separation estimated and showed on the fluid precious stone with the assistance of the regulator. The regulator gets the sign and it with a hand-off utilizing triggers firearm.

- $\quad$ The sensor fitted over the radio wire with the stepper engine pivots 360 degrees.

- If there is any rocket or item close by the frame work, at that point it dispatches hostile to rocket to the objective article.

- The tank vehicle will likewise be having another regulator which is utilized to control the vehicle.

- Mp lab ide programming utilized by the regulator when the rocket isn't distinguishing inside range. When there is a benevolent item close by, this product decides it as a companion rocket

Figure 5: Working model of missile detection and destroy system

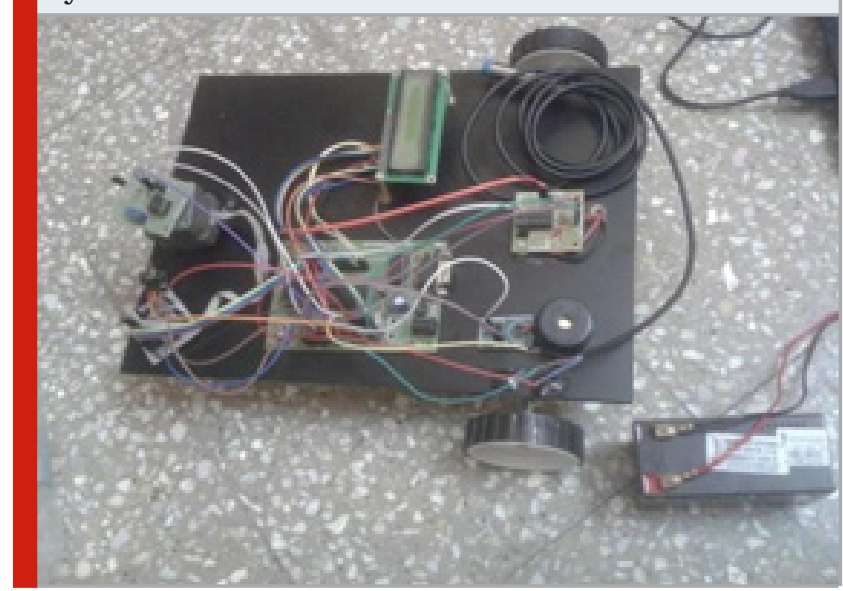

\section{RESULTS AND DISCUSSION}

We can locate the rocket by using ultrasonic waves and then start the destroyer with the smaller scope regulator. In different watchman areas, this can be implemented to defend the nation from new attacks. The operation of antimissile surveillance ends up becoming notable in the event of a general military conflict.Yet monster attempts are put into this region against rocket watch with a hundred percent faithful quality was not reached by any country. Therefore, identifiable evidence systems, controllable missiles, high-power lasers are used in the early ambush. We should understand that such innovations will never be used for their rapid goal, 
but will never be applied for peaceful purposes by mankind.

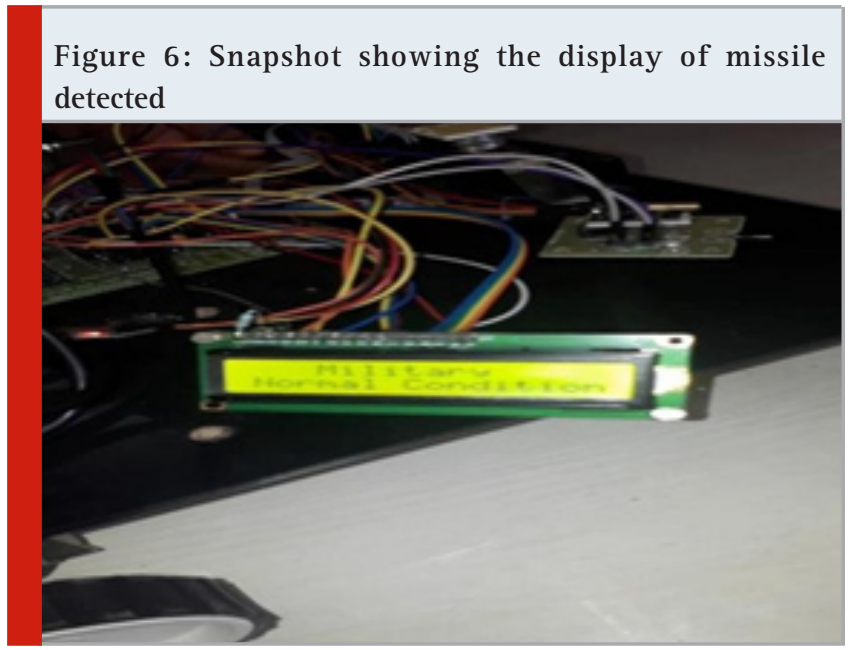

Table 1. Comparison analysis of the proposed and existing method

\begin{tabular}{|l|c|c|}
\hline Methods & Precision (\%) & Recall (\%) \\
\hline DeepSqueak [19] & 82 & 86 \\
\hline Proposed & 87 & 91 \\
\hline
\end{tabular}

Figure 7: Comparison analysis of proposed and existing method in detection

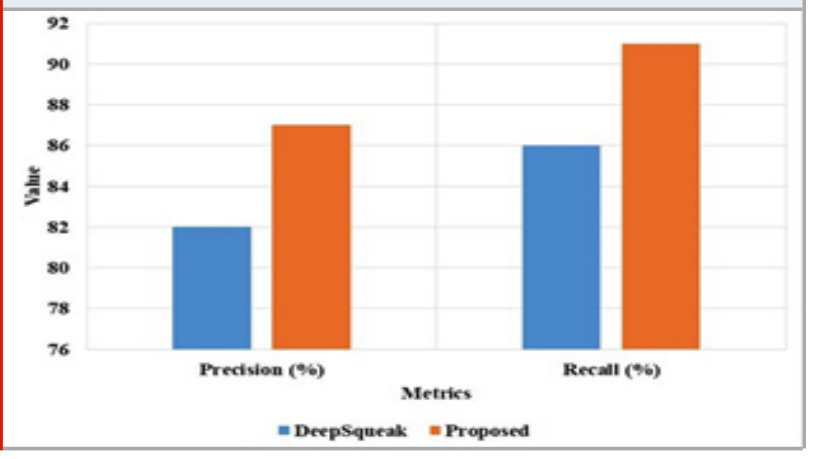

The circuit is used to receive $40 \mathrm{KHz}$ reflected signals from the missile object, to feed them to the microcontroller program and to turn on the required load while the microcontroller is running the program. When the microcontroller receives the signal from the ultrasonic receiver, it triggers the door gun via a transistor or relay by activating the MOSFET gate. The sensor is mounted on the antenna and is rotated and operated by a 360 degree stepper motor.The program will move the launcher to the nearest detected target and fire if there is any target inside the detection range.

The proposed method is compared with the existing method in the detection process in terms of precision and recall, as shown in Table 1 and Figure 7. The result shows that the proposed method has higher performance in detection compared to DeepSqueak method. The proposed method has $87 \%$ precision and existing DeepSqueak method has $82 \%$ precision in detection.

\section{CONCLUSION}

The task "Rocket Discovery and Decimating Framework" has been effectively structured and tried. Coordinating highlights of all the equipment segments utilized have created it. Nearness of each module has been contemplated out and put cautiously in this way adding to the best working of the unit. Furthermore, utilizing profoundly propelled IC's and with the assistance of developing innovation the task has been effectively actualized. Finish of our exploration is by utilizing ultrasonic sensor with stepper engine we persistently identify the objective toward all path. Furthermore, if and is separated ultrasonic beams by utilizing laser shaft we show that target is obliterate however in real framework by utilizing unique kind of firearm or rocket we will crush target totally and Automated stage is helpful for the development of entire circuit conceivable way. This undertaking of rocket discovery and selfsufficient propelling framework is adjusted to focus on a rocket object in three-hub revolution by following the Ultrasonic handset information. Here we develop a protection system which can protect the cruise missile from the threat of anti-cruise missiles. The advantage of this system is that the whole thing can be done with a small cost.

Here we do not need to develop any external tracking system, the cruise missile itself carry the tracking system and itself able to take decision. But we can also make arrangement for giving manual command or directions in this regard. This system provide protection during its whole journey period of the cruise missile, this is from launching to attack. This kind of protection system can also be developed for other type of missiles. In future with the more advanced devices we are able to design more reliable and accurate protection system for the cruise missile.

Future Scopes: We can likewise utilize diverse programming and symbolism for discovery purposes. More innovations can be utilized for building up an enemy of rocket strategy with a similar working rule.

We will grasp the sharp structure in the future by using the regulator microcontroller.

In the future, it will usually be used as a globally moved framework close to a high-power camera to meet a true target (say a Rocket or Tank).

The benefit of this device is that we can use camcorders and different sensors to see the live target moving from anywhere on the planet in order to operate the structure. In the future, along with a high-intensity camera, it can be used as an advanced tracking device to track a real target (say a missile or tank). The benefit of this unit is that we can use video camera and other sensors to see the live moving target from anywhere in the world to operate the system. 


\section{REFERENCES}

Chai, R., Savvaris, A. and Chai, S., 2019. Integrated missile guidance and control using optimization-based predictive control. Nonlinear Dynamics, 96(2), pp.9971015.

Coffey, K.R., Marx, R.G. and Neumaier, J.F., 2019. DeepSqueak: a deep learning-based system for detection and analysis of ultrasonic vocalizations. Neuropsychopharmacology, 44(5), pp.859-868.

Garcia, E., Casbeer, D.W., Fuchs, Z.E. and Pachter, M., 2017. Cooperative missile guidance for active defense of air vehicles. IEEE Transactions on Aerospace and Electronic Systems, 54(2), pp.706-721.

Guo, J., Xiong, Y. and Zhou, J., 2018. A new sliding mode control design for integrated missile guidance and control system. Aerospace Science and Technology, 78, pp.54-61.

Hong, D., Kim, M. and Park, S., 2020. Study on Reinforcement Learning-Based Missile Guidance Law. Applied Sciences, 10(18), p.6567.

Kumar, S. and Furuhashi, H., 2017. Long-range measurement system using ultrasonic range sensor with high-power transmitter array in air. Ultrasonics, 74, pp.186-195.

Kumar, S. and Furuhashi, H., 2018. Characteristics of an ultrasonic phased array transmitter in medium range. Ultrasonics, 82, pp.331-335.

M. Anushree Kirthika, "missile detection and automatic destroy system ",Advances in Aerospace Science and Applications ISSN 2277- 3223 volume 4, number 1(2014),pp.16.

Ming, C., Wang, X. and Sun, R., 2019. A novel nonsingular terminal sliding mode control-based integrated missile guidance and control with impact angle constraint. Aerospace Science and Technology, 94, p.105368.

Missile Detection by Ultrasonic and Auto Destroy System" by Samir Chopra, published on International Journal of Engineering] Sciences\&t Research Technology.

Praveen Kumar, "IoT Based Monitoring and Control of Appliances for Smart Home”, in IEEE International
Conference on Recent Trends in Electronics Information Communication Technology, pp.1145-1150, 2016.

Puttamadappa, C. and Parameshachari, B.D., 2019. Demand side management of small scale loads in a smart grid using glow-worm swarm optimization technique. Microprocessors and Microsystems, 71, p.102886.

Samir chopra, Suman bharati, Tarun singh negi, Prof. Ms. P.D. Kulkarni, "Missiledetection by ultrasonic and auto destroy system", international journal ofengineering science and research technology may (2014).

Shibani S. Raikar, "short range missile tracking system”, international journal of scientific and technology research volume 2, issue 9, September 2013.

Suchitha Samuel, B.Bharathi, J.Mrudula, Department of Electronics\&t Communication Engineering, Geethanjali College of Engineering and Technology, Keesara, Andhra Pradesh, India, Design of PC Controlled Automatic Solar Tracker Robot, Vol. 2, Issue 10, October 2013, International Journal of Innovative Research in Science, Engineering and Technology.

Wang Shaokun, Xiao Xiao, Zhao Hongwei The Wireless Remote Control Car System Based On ARM9, College of Software, Department of Computer Science and Technology, Jilin University, JLU, Changchun, China, 2011 International Conference on Instrumentation, Measurement, Computer, Communication and Control.

Wang, X., Zhao, H., Han, T., Wei, Z., Liang, Y. and Li, Y., 2019. A Gaussian estimation of distribution algorithm with random walk strategies and its application in optimal missile guidance handover for multi-UCAV in over-the-horizon air combat. IEEE Access, 7, pp.4329843317.

Weiss, M. and Shima, T., 2018. Linear quadratic optimal control-based missile guidance law with obstacle avoidance. IEEE Transactions on Aerospace and Electronic Systems, 55(1), pp.205-214.

Zhang, W. and Yi, W., 2020. Fuzzy Observer-Based Dynamic Surface Control for Input-Saturated Nonlinear Systems and its Application to Missile Guidance. IEEE Access, 8, pp.121285-121298. 